\title{
Disposition des enseignants marocains à l'adoption des ressources éducatives libres: état des lieux et perspectives
}

\section{Disposition of Moroccan teachers towards the adoption of open educational resources: state of art and perspectives}

\author{
Mourad BENALI ${ }^{1,2 *}$, Mohammed BOUKARE ${ }^{2}$, Samira ELOUELJI ${ }^{2}$ and Mohammed \\ BLEJ $^{1}$ \\ ${ }^{1}$ Centre régional des métiers de l'éducation et de la formation, Région de l'oriental, Oujda, Maroc \\ ${ }^{2}$ Laboratoire CEDUC, FLSHO, Université Mohammed I, Oujda, Maroc
}

\begin{abstract}
Résumé. Les systèmes éducatifs à l'échelle mondiale explorent le potentiel des Ressources Educatives Libres (REL). Cependant, l'adoption des REL reste limitée chez les enseignants. Cette étude exploratoire s'appuie sur le modèle pyramidal d'adoption des REL et a pour objectif de recueillir des renseignements de base sur le niveau de connaissance et d'utilisation des REL. Un questionnaire en ligne a été mis à la disposition de 1297 enseignants marocains. Les résultats révèlent une méconnaissance du concept de REL, des sources où l'on peut les trouver et des cadres d'octroi de licences et de droits d'auteurs chez la majorité des répondants. L'inclusion de l'adoption des REL dans les modules de formation des enseignants est fortement recommandée.
\end{abstract}

\begin{abstract}
Education systems worldwide are exploring the potential of Open Educational Resources (OER). However, the adoption of OER among teachers remains limited. This exploratory study builds on the pyramid model of OER adoption and aims to collect baseline information on the level of knowledge and use of OER. An online questionnaire was made available to a group of Moroccan teachers $(n=1297)$. The results a lack of awareness of the concept of OER and the sources where to find them, and of licensing and copyright frameworks is widely observed among the majority of respondents. The inclusion of OER adoption in teacher training modules is strongly recommended.
\end{abstract}

\footnotetext{
* Corresponding author: m1.benali@ump.ac.ma
} 


\section{Introduction}

Face à la croissance rapide du taux de scolarisation, à l'augmentation limitée des ressources financières consacrées au secteur de l'éducation, le soutien organisé et volontaire apporté aux systèmes éducatifs revêt une importance accrue afin d'améliorer les outils d'enseignement et d'apprentissage et élaborer des outils d'évaluation efficaces ainsi que déterminer les moyens de répondre aux besoins des apprenants dans leur diversité grandissante. La qualité des résultats des apprentissages de chaque apprenant dépend de nombreux facteurs au rang desquels la possession des manuels scolaires requis ainsi que d'autres matériels didactiques [1].

Avec le développement des technologies de l'information et de la communication (TIC) et surtout du Web, les créateurs de contenus éducatifs se mettent de plus en plus à les partager librement sur internet. Cette notion de partage de contenus est connue sous le nom de contenu ouvert ou l'Open Content en anglais. Plusieurs organisations se sont donc inspirées de ce mouvement du contenu ouvert, en particulier les organisations éducatives qui ont commencé depuis 1990 à mettre en place du matériel pédagogique et à le partager librement sur internet. L'expression « Ressources Educatives Libres (REL) » a été adoptée pour la première fois en 2002 lors du forum organisé à Paris par l'UNESCO en coopération avec la Fondation William and Flora Hewlett sur l'impact des didacticiels libres pour l'enseignement supérieur dans les pays en développement : "Les participants ont ensuite adopté une Déclaration finale dans laquelle ils "expriment leur satisfaction et leur volonté de développer ensemble des ressources éducatives universelles disponibles pour toute l'humanité, qui s'appelleront dorénavant Ressources Educatives Libres" " [2].

Les ressources éducatives libres, ou REL, s'inscrivent directement dans la philosophie de l'apprentissage ouvert. Le terme fait globalement référence à des documents éducatifs accessibles sans frais et dont le partage et la reproduction sont permis sans avoir à payer de droits d'auteur. Il s'agit en général de documents numérisés, donc ils sont libres d'accès dans la mesure où l'utilisateur dispose d'un accès au Web.

En 2012 et lors de la déclaration de Paris, l'UNESCO a énoncé une définition plus raffinée des REL: "Les ressources éducatives libres (REL) sont des matériaux d'enseignement, d'apprentissage ou de recherche appartenant au domaine public ou publiés avec une licence de propriété intellectuelle permettant leur utilisation, adaptation et distribution à titre gratuit».

Les REL peuvent donc comprendre des cours/programmes complets, cours de formation, modules, guides de l'étudiant, notes pédagogiques, manuels, articles de recherche, vidéos, outils et instruments d'évaluation, matériels interactifs tels que simulations, jeux de rôle, bases de données, logiciels, applications (notamment applications mobiles) et tout autre matériel utile à des fins éducatives.

Un ensemble de cinq permissions connues sous le sigle de $5 \mathrm{R}$ déterminent le caractère libre ou non d'une ressource éducative, à savoir les droits de Retenir (télécharger et conserver une ou des copies), Réutiliser (la ressource à ses propres fins), Réviser (modifier), Remixer (avec d'autres ressources) et Redistribuer (partager la ressource, modifiée ou non) [3]. Ces caractéristiques offrent aux enseignants des avantages pédagogiques leur permettant d'adapter les ressources à leurs besoins pédagogiques spécifiques [4]. D'autres recherches confirment que les enseignants qui ont adopté ce genre de ressources sont devenus plus innovants dans leurs pratiques pédagogiques, grâce à l'ouverture et à la flexibilité permises par les licences ouvertes [5-7]. L'UNESCO a adopté en 2019 une recommandation spécifique concluant que les REL sont estimées fournir les outils nécessaires à l'atteinte des objectifs de développement durable (ODD) 2030 des Nations Unies [8].

À l'échelle internationale, le développement des REL a donné lieu à de multiples initiatives et projets de nature institutionnelle dont : 
- La publication d'un référentiel de compétences par l'Organisation Internationale de la Francophonie (OIF) en 2016 afin de transmettre les quatre compétences fondamentales que sont la recherche, la réutilisation, la conception et la diffusion des REL ;

- Le moteur national d'indexation de REL en France ;

- L'initiative IDNEUF portée par l'Agence Universitaire de la Francophonie (AUF). Avec plus de 11,6 millions de références documentaires libres d'accès dans tous les domaines des sciences et de la culture ;

- Le portail de ressources californien MERLOT4 (Multimedia Educational Resource for Learning and On-Line Teaching);

- La coalition internationale SPARC (Scholarly Publishing and Academic Resources Coalition);

- Le réseau mondial de l'Open Education Consortium ;

- ORBIT: The Open Resource Bank for Interactive Teaching. Initiée par la faculté d'éducation, Université de Cambridge.

- L'initiative pour la formation des enseignants en Afrique subsaharienne (TESSA) initiée et lancée en 2010 par l'Open University britannique ;

- L'initiative de l'Université Virtuelle Africaine "REL@UVA". Lancée en 2011, en juillet 2018, REL@UVA comptait un total de 1623 de REL.

- Fabrique REL pour la conception et la production de REL. Il s'agit d'un projet conjoint de l'Université de Sherbrooke, de l'Université de Montréal et de l'Université Laval, réalisé dans le cadre d'un mandat octroyé en 2019 par le Ministère de l'Éducation et de l'Enseignement Supérieur et du Plan d'action numérique en éducation et en enseignement supérieur au Québec.

Cependant, malgré ce contexte potentiellement fédérateur et prometteur, les REL peinent à se développer dans les usages pédagogiques des enseignants. Ce constat est confirmé par un ensemble d'études. Les raisons évoquées sont multiples selon les variables suivantes :

Table.1. Obstacles d'adoption des REL selon les études.

\begin{tabular}{|l|l|}
\hline Variables & \multicolumn{1}{|c|}{ Études } \\
\hline $\begin{array}{l}\text { Manque de stratégies institutionnelles au sein des } \\
\text { établissements pour valoriser et encourager la réutilisation et le } \\
\text { partage des REL par leurs enseignants. }\end{array}$ & {$[13] ;[10] ;[11] ;[12] ;$} \\
\hline $\begin{array}{l}\text { Sensibilisation et formation des enseignants à l'adoption des } \\
\text { REL dans leurs pratiques. }\end{array}$ & $\begin{array}{l}{[14] ;[10] ;[9] ;[15] ;} \\
[11] ; 16] ;[4] ;[13] ;\end{array}$ \\
\hline $\begin{array}{l}\text { Qualité variable des ressources et manque de confiance dans les } \\
\text { sources exploitées par les REL. }\end{array}$ & {$[13] ;[18] ;[17]$} \\
\hline $\begin{array}{l}\text { Raisons intrinsèques liées aux valeurs et convictions des } \\
\text { enseignants (préférence pour créer ses propres ressources, } \\
\text { crainte du mésusage). }\end{array}$ & {$[10] ;[9] ;[19] ;[13]$} \\
\hline $\begin{array}{l}\text { Ressources crées pour un usage pédagogique trop fortement } \\
\text { contextualisé ou personnalisé (selon les besoins d'un auteur- } \\
\text { concepteur et non d'un public potentiellement plus large). }\end{array}$ & {$[20] ;[21]$} \\
\hline $\begin{array}{l}\text { Difficultés liées aux technologies, aux plateformes, à la } \\
\text { localisation ou à l'indexation des REL. }\end{array}$ & {$[22] ;[23] ;[9] ;[13]$} \\
\hline Méconnaissance et incertitudes envers les licences ouvertes. & {$[9] ;[13] ;[24]$} \\
\hline Manque de temps. & {$[9] ;[25]$} \\
\hline
\end{tabular}


$\mathrm{Au}$ niveau local, la société marocaine connaît une croissance soutenue de la population urbaine, une augmentation continue des besoins de scolarisation et une plus grande aspiration des familles à une éducation de qualité pour leurs enfants, dans le cadre d'une société qui attend efficacité et équité de son système éducatif. Le Forum National sur la stratégie des REL au Maroc tenu en décembre 2016 à l'université Cadi Ayyad à Marrakech a constitué le point de départ de la Déclaration du Maroc sur les REL. Cette déclaration appelle les autorités marocaines et tous les secteurs de l'éducation marocaine à approuver un ensemble de recommandations dont les principales sont:

- Favoriser la sensibilisation à toutes les formes d'éducation ouverte dans tous les secteurs de l'éducation marocaine.

- Promouvoir les pratiques d'éducation ouverte et l'utilisation des ressources éducatives libres pour élargir l'accès à l'apprentissage tout au long de la vie à tous les niveaux.

- Encourager l'utilisation de licences ouvertes (par exemple, Creative Commons) pour tous les documents éducatifs produits avec des fonds publics, permettant différents types d'utilisation, tout en respectant les droits des détenteurs de droits d'auteur.

- Soutenir le développement d'outils TIC conviviaux pour trouver, récupérer et partager des ressources d'éducation ouverte qui sont pertinentes pour les besoins des enseignants et des apprenants, en fonction de normes ouvertes afin d'assurer l'interopérabilité et la réutilisation des cours ouverts, des ressources et du matériel d'évaluation.

Devant ce contexte international, l'usage des REL est devenu une préoccupation importante pour un grand nombre d'institutions académiques. Vu le déficit de recherches nationales empiriques relatives à ce sujet, nous avons considéré le besoin d'explorer les niveaux de connaissance et d'usage des REL chez les enseignants marocains exerçant dans l'enseignement scolaire. Le contenu de notre article est organisé en trois parties : une première consacrée à la présentation du cadre conceptuel régissant les facteurs d'adoption des REL. La seconde est méthodologique où nous allons expliciter le mode de sélection du corpus et la finalisation de l'instrument de collecte des données. La dernière partie permettra de présenter et discuter les résultats.

\section{Cadre théorique}

Dans le cadre de notre étude, nous nous appuyons sur la pyramide d'adoption des REL élaborée par Cox et Trotter en 2016. Cette dernière s'inspire de la hiérarchie des besoins de Maslow [29] et utilise une approche analytique à plusieurs niveaux. La pyramide d'adoption des REL présentée dans la figure 1 regroupe les facteurs essentiels d'adoption des REL en six catégories, classées selon le niveau de contrôle que chaque enseignant y exerce. De la détermination externe à la détermination interne, ces facteurs sont : l'accès aux infrastructures, la permission légale, la sensibilisation, la compétence technique, la disponibilité des ressources et la volonté individuelle. 


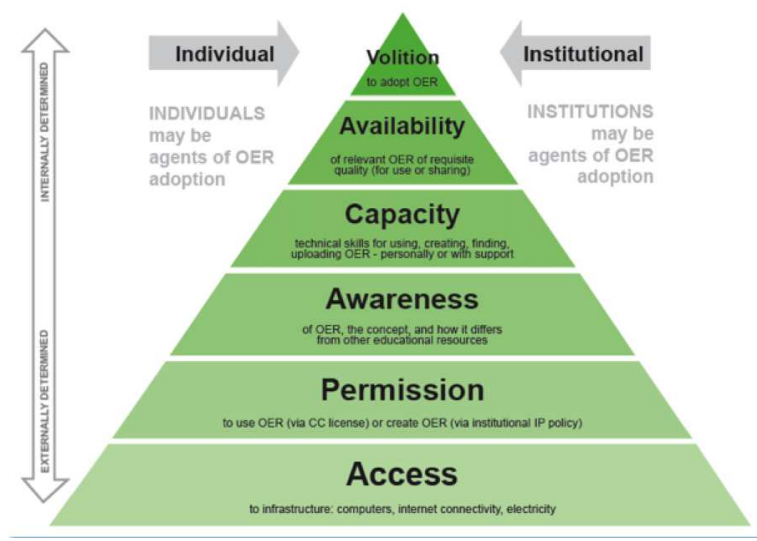

Fig. 1. Pyramide d'adoption des REL [30].

La pyramide représente graphiquement six gradations catégorielles dans un spectre d'interdépendance de facteurs externes-internes relatifs à un usage individuel ou institutionnel. Dans notre étude, nous nous intéressons au volet d'adoption individuel comme suit :

- Accès (Access) : Le premier facteur concerne l'accès des enseignants à l'infrastructure physique et au matériel approprié, tels que l'électricité, la connectivité et les dispositifs informatiques nécessaires pour utiliser les REL à caractère numérique.

- Permission (Permission): Le deuxième facteur porte d'une part sur l'accord d'adoption des REL par les institutions en conformité avec leur politique de la propriété intellectuelle. D'autre part, il concerne la connaissance par les enseignants des dispositions de licence d'utilisation et des droits d'auteur au regard de l'adoption des REL.

- Sensibilisation (Awareness) : Le troisième facteur explore le degré de connaissance du concept des REL et en quoi elles diffèrent des autres ressources éducatives (généralement soumis à des droits d'auteur). Les enseignants peuvent bien sûr utiliser les REL par inadvertance, ce qui exige un certain niveau de sensibilisation.

- Capacité (Capacity) : Le quatrième facteur concerne les compétences numériques et sémantiques nécessaires à l'adoption des REL. Ces compétences peuvent être détenues par l'enseignant ou mobilisées grâce à un soutien institutionnel. Cela implique qu'un enseignant jouit de la fluidité technique nécessaire pour rechercher, identifier, utiliser et/ou créer (licence et téléchargement) des REL, ou bénéficie du soutien de personnes ressources possédant ces compétences. Si les enseignants connaissent les REL, ils doivent posséder des compétences techniques pour les trouver, les utiliser, les créer et les télécharger. Le manque de connaissance des droits de propriété intellectuelle et des licences ouvertes a une influence négative sur l'utilisation des REL par les enseignants [24].

- Disponibilité (Availability) : Le cinquième facteur concerne la disponibilité des REL pour qu'un enseignant puisse les utiliser ou contribuer à leurs développements. Ce facteur est déterminé par la pertinence des REL (contenu, portée, niveau, langue, format), l'utilité pour leurs utilisations spécifiques prévues et leurs qualités.

- Volonté (Volition) : Le dernier facteur d'adoption des REL est lié à la motivation des enseignants à adopter les REL. Elle est fortement dépendante et influée par :

- Les autres facteurs du modèle pyramidal à savoir : l'accès, la permission, la prise de conscience, le savoir-faire et la disponibilité.

- Le contexte social (normes départementales et disciplinaires) et les structures institutionnelles (politiques, stratégies et mécanismes).

- Les croyances et pratiques personnelles, idiosyncrasiques et internes. En l'occurrence, le style d'enseignement (interactif, traditionnel ;...), la philosophie de 
l'enseignant au regard de l'éducation, son niveau d'estime de ses propres ressources pédagogiques ([26] ; [27] ; [28]), sa capacité émotionnelle concernant le jugement porté par d'autres personnes sur son travail, etc.

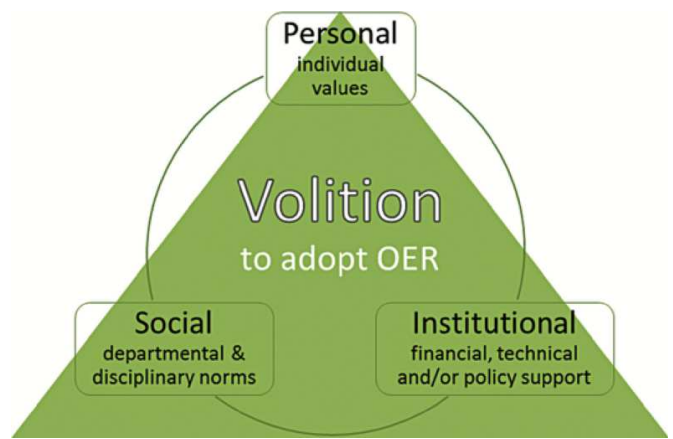

Fig. 2. Variables de volonté dans la pyramide d'adoption des REL [31].

Un ensemble de variables à associer à chaque facteur ont été identifiés et sont présentés dans le tableau suivant.

Tab. 2. Variables associées à chaque facteur de la pyramide d'adoption des REL.

\begin{tabular}{|c|c|}
\hline Facteurs & Variables associées \\
\hline Volonté & $\begin{array}{l}\text { - Style d'enseignement ; } \\
\text { - Philosophie de l'éducation; } \\
\text { - Niveau de confiance en soi dans l'usage de ses ressources pédagogiques } \\
\text { dans l'enseignement; } \\
\text { - Incitation et reconnaissance institutionnelles; } \\
\text { - Contexte social : normes départementales, disciplinaires et collégiales } \\
\text { concernant l'utilisation des REL ; } \\
\text { - Considérations relatives aux coûts et aux inconvénients d'usage des REL } \\
\text { - Conséquences temporelles de l'usage des REL; }\end{array}$ \\
\hline Disponibilité & $\begin{array}{l}\text { Disponibilité en relation avec: } \\
\text { - La qualité des REL (exactitude, exhaustivité, rigueur); } \\
\text { - La pertinence des REL (en termes de perspectives épistémiques, de } \\
\text { portée, de langue, de format, de localisation, etc.); } \\
\text { - L'utilité des REL (dans un objectif pédagogique spécifique et anticipé). }\end{array}$ \\
\hline Capacité & $\begin{array}{l}\text { - Savoir-faire technique en matière de recherche, identification, } \\
\text { téléchargement, } \\
\text { utilisation (réutilisation, édition, remixage) et partage des REL. }\end{array}$ \\
\hline Sensibilisation & $\begin{array}{l}\text { - Compréhension conceptuelle des REL; } \\
\text { - Compréhension conceptuelle des différentes licences et droits d'auteurs. }\end{array}$ \\
\hline Permission & $\begin{array}{l}\text { - Politiques de propriété intellectuelle (institutionnel); } \\
\text { - Politiques en matière de droits d'auteur- copyright } \\
\text { (national/institutionnel); } \\
\text { - Paramètres de la licence ouverte appliquée aux REL. }\end{array}$ \\
\hline Accès & $\begin{array}{l}\text { - Accès à Internet ; } \\
\text { - Accès aux équipements informatiques ; } \\
\text { - Accès à l'électricité. }\end{array}$ \\
\hline
\end{tabular}

Dans le cadre de notre étude, nous nous appuyons sur la pyramide d'adoption des REL. Ce modèle analytique est un point de départ utile, surtout lorsque l'analyse porte sur des contextes dans lesquels l'activité REL est dans une phase embryonnaire. En d'autres termes, 
ce modèle garde délibérément une vision étroite sur les facteurs qui doivent être pris en considération pour que des activités d'adoption des REL se manifestent et s'installent.

\section{Méthodologie}

\subsection{Population de l'étude}

Notre démarche méthodologique repose sur deux approches à savoir une approche quantitative basée sur une enquête par questionnaire visant à établir une vue d'ensemble des facteurs du modèle pyramidal. Cette enquête auprès d'un groupe d'enseignants marocains a été réalisée par le biais d'un questionnaire en ligne. Ce dernier a été diffusé au courant de l'année académique 2018-2019 et plus particulièrement en mois de février 2019 auprès d'enseignants relevant de niveaux et disciplines confondus.

Le recrutement de notre échantillon d'enseignants a été effectué à travers des divers canaux informels de communication, y compris des groupes d'enseignants sur Facebook et WhatsApp, les listes de diffusion de réseaux professionnels et de communautés de pratiques d'enseignants et les différentes associations professionnelles d'enseignants.

\subsection{Instrument de collecte des données}

La construction de l'instrument de collecte de données a constitué une étape importante afin que celui-ci correspond le plus possible aux objectifs de l'étude. Notre outil de mesure est une adaptation issue principalement de plusieurs questionnaires [1,9]. La différence des contextes d'application et le changement de l'amplitude des échelles de mesure requéraient l'adaptation de notre questionnaire au contexte éducatif marocain.

Dans un premier temps, ce questionnaire a été mis à l'essai auprès d'un groupe restreint d'enseignants pour confirmer que les questions et la terminologie étaient conformes pour des fins d'optimisation de notre instrument de collecte de données. Les concepts du sondage étaient reliables au niveau du test de fiabilité. En effet, le coefficient Alpha de Cronbach variait entre 0,72 et 0,77 . Les modalités de réponse ont été adaptées pour chaque question figurant dans le questionnaire. Ce dernier comprend trois sections. Une première consacrée à la description de notre échantillon, une deuxième portant sur l'accès aux équipements technologiques et la troisième composée par un ensemble d'items correspondant aux facteurs du modèle pyramidal.

Afin d'avoir une compréhension homogène et une connaissance de cause des REL, une définition de ces ressources a été fournie aux répondants qui devaient aborder les items portant sur les facteurs : permission, sensibilisation, capacité et disponibilité. L'analyse des données quantitatives a compris des processus sur Excel pour obtenir des statistiques principalement descriptives.

\section{Résultats}

\subsection{Description de l'échantillon}

$\mathrm{Au}$ total, 1297 participants ont répondu au sondage. Globalement, notre échantillon comprend $64 \%$ d'hommes et $36 \%$ de femmes. L'échantillon étudié présente aussi une dominance de la catégorie d'âge inférieur à 41 ans, à hauteur de $81 \%$.

$\mathrm{Au}$ regard des années exercées dans l'enseignement et des niveaux enseignés de notre échantillon, les éléments recueillis dans le cadre du sondage confirment que la globalité des 
répondants sont majoritairement issus de l'enseignement scolaire (primaire, secondaire collégial et secondaire qualifiant).

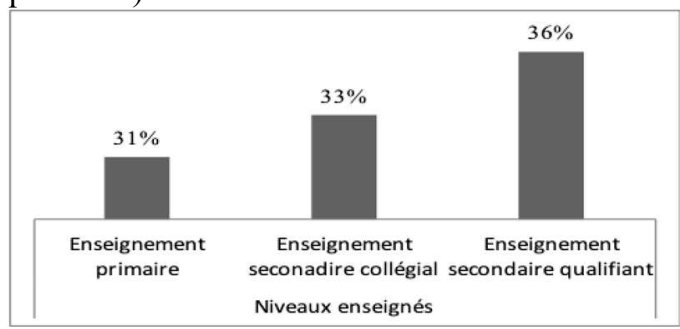

Fig. 3. Proportions globales de l'échantillon étudié selon le niveau enseigné.

Au niveau des années d'expérience dans l'enseignement, 75\% de notre population d'étude sont de nouveaux recrus et ont moins de 10 années d'expérience dans l'enseignement.

\section{Années d'expérience dans l'enseignement}

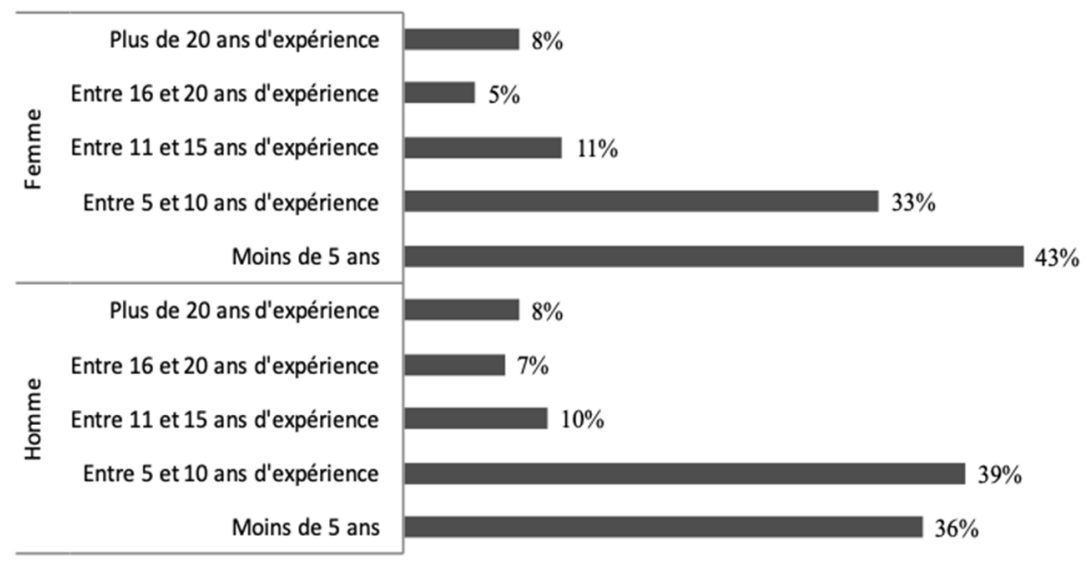

Fig. 4. Proportions globales de l'échantillon étudié selon les années d'expérience dans l'enseignement.

\subsection{Disposition des enseignants à l'adoption des REL}

\subsubsection{Accès}

Dans un premier temps, nous avons questionné les participants au sujet de leur accès aux équipements technologiques. Les résultats affichent de très importantes proportions d'équipements technologiques (figure 5).

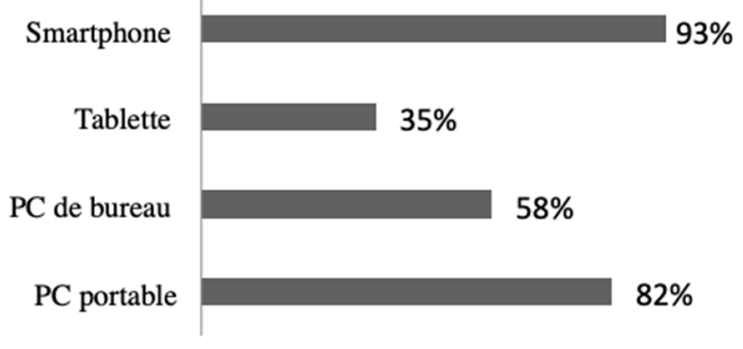


Fig. 5. Proportions globales d'accès aux dispositifs technologiques

Au regard de la fréquence de connexion à Internet, les résultats révèlent des taux importants (journaliers et par semaine) en matière d'accès à Internet chez notre population d'étude (Figure 6).
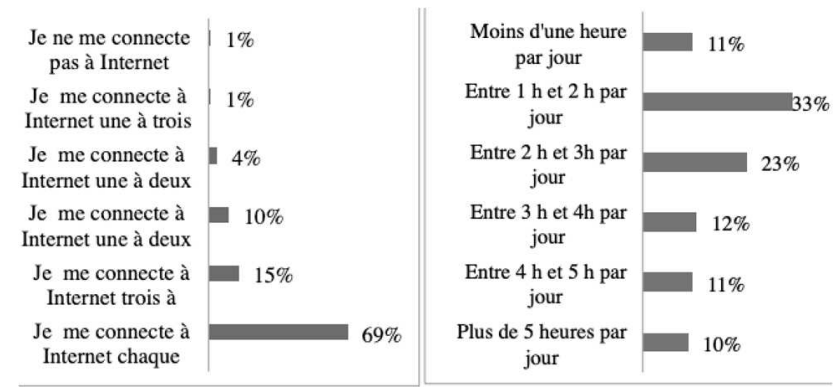

Fig. 6. Fréquence d'accès à Internet chez l'échantillon d'étude

Au niveau des lieux de connexion à Internet chez les répondants, les résultats montrent que le domicile reste le lieu majoritaire de connexion à internet (Figure 7).

$84 \%$

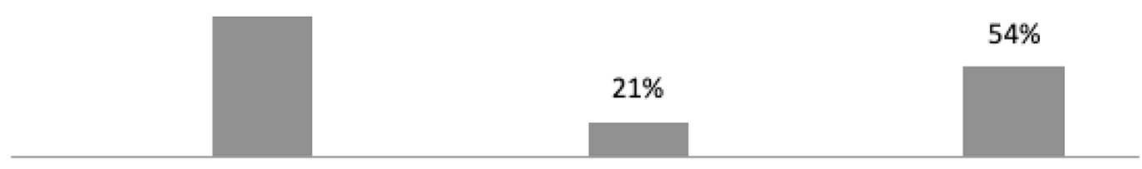

Je me connecte depuis le Je me connecte depuis une Je me connecte via dans domicile connexion fournie par d'autres lieux, tels que les l'établissement scolaire cafés, les lieux public et autres

Fig. 7. Lieux de connexion à Internet chez les enseignants.

On a également demandé aux participants d'apprécier leur niveau d'aisance en l'usage des Technologies d'Information et de Communication (TIC) à des fins d'enseignement. $53 \%$ des interrogés déclarent être confortables en l'usage de ces ressources à des fins d'enseignement, ce qui traduit une banalisation moyenne des pratiques numériques.

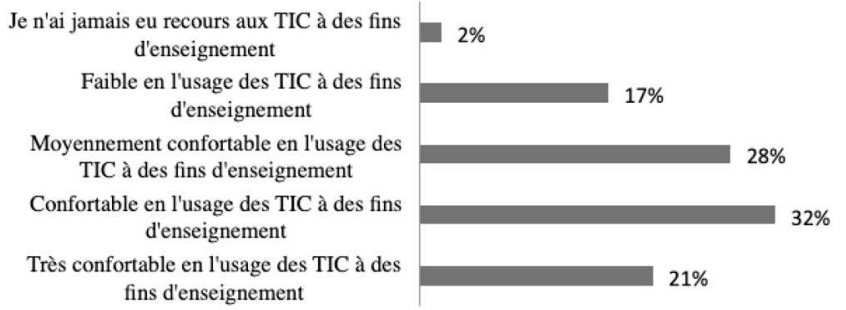

Fig. 8. Niveau d'aisance en l'usage des TIC à des fins d'enseignement.

\subsubsection{Permission}

Dans un premier temps, nous avons demandé à notre population d'étude de spécifier l'entité responsable de la sélection et l'usage des ressources d'enseignement/apprentissage dans la liste suivante : 
- Je suis seul responsable de la sélection et l'usage des ressources que j'utilise dans mes pratiques d'enseignement/apprentissage ;

- Je suis membre d'un comité qui fait la sélection et l'usage des ressources que j'utilise dans mes pratiques d'enseignement/apprentissage ;

- L'inspecteur pédagogique est responsable de la sélection et l'usage des ressources des ressources que j'utilise dans mes pratiques d'enseignement/apprentissage ;

- Il existe des directives officielles concernant la sélection et l'usage des ressources que j'utilise dans mes pratiques d'enseignement/apprentissage.

Quant aux rôles dans la sélection et l'usage des ressources d'enseignement, $83 \%$ des répondants déclarent avoir le plein contrôle sur les décisions relatives à la sélection et l'usage des ressources dans leurs pratiques d'enseignement/apprentissage. 31\% des enseignants seulement approuvent les décisions prises en collaboration au sujet des ressources des cours (Figure 9).

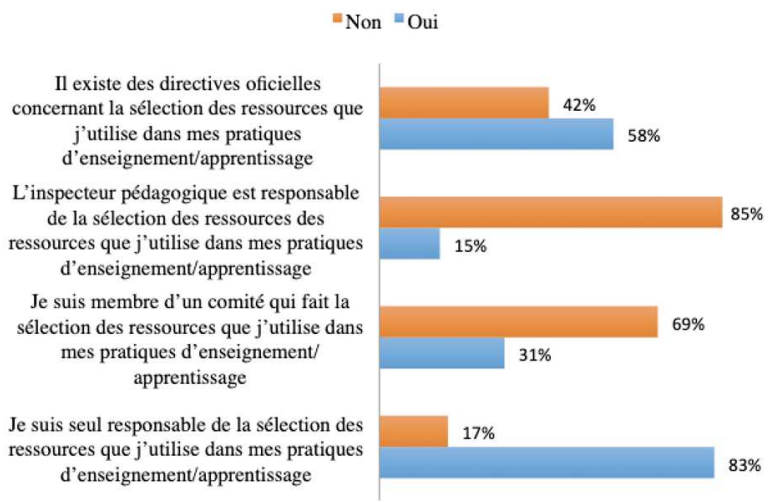

Fig. 9. Rôles dans la sélection et l'usage des ressources d'enseignement.

La figure suivante concerne les déterminants potentiels de sélection des ressources que les enseignants utilisent dans leurs pratiques d'enseignement. Les enseignants sont invités à indiquer leurs préférences dans la liste suivante en sélectionnant un choix entre « Entièrement d'accord, D'accord, Neutre, En désaccord ou Fortement en désaccord ».

- La ressource du cours est gratuite ;

- La ressource du cours contient du contenu complet et des activités d'apprentissage ;

- La ressource du cours est recommandée par des collègues enseignants ;

- La ressource du cours est recommandée par l'inspecteur pédagogique ;

- La ressource du cours est riche en médias (illustrations, vidéos, animations, simulations);

- La ressource du cours comprend des documents supplémentaires (diapositives PowerPoint, banques de questions) ;

- La ressource du cours est disponible en formats accessibles.

Comme le montre la figure, les trois principaux déterminants de sélection ressources d'enseignement chez les enseignants portent en général sur le contenu et le format de ces ressources selon : 
- La richesse en médias à savoir : illustrations, vidéos, animations, simulations ;

- La richesse du cours en un contenu complet et des activités d'apprentissage ;

- La disponibilité du cours en formats accessibles.

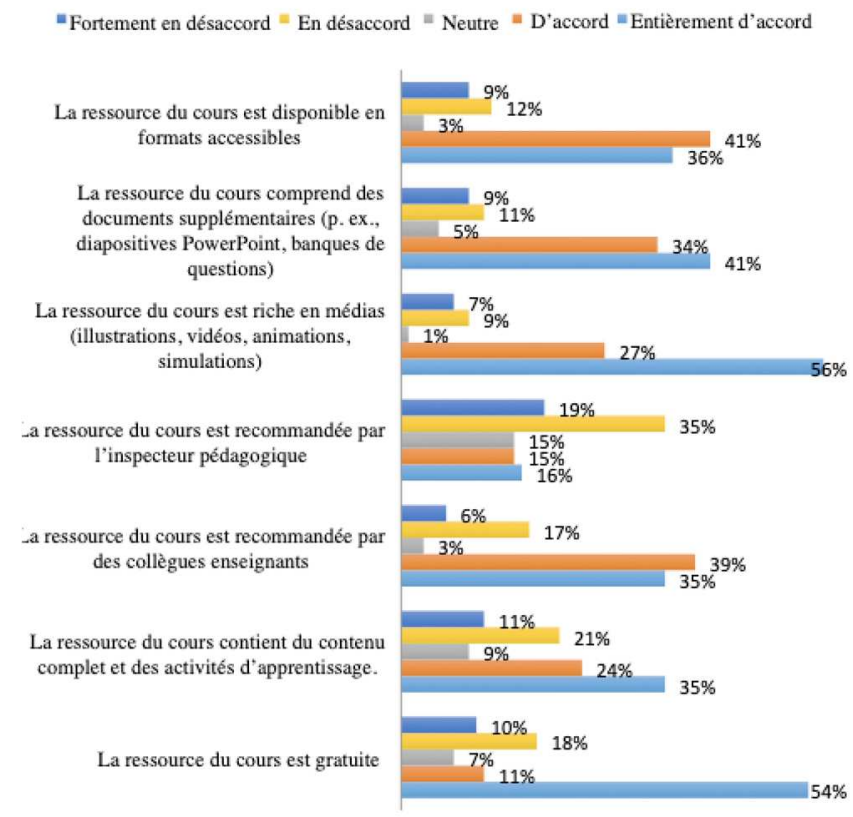

Fig. 10. Déterminants de sélection des ressources d'enseignement chez les enseignants.

Enfin, lorsqu'on a demandé aux enseignants de notre échantillon d'indiquer les types de ressources qu'ils utilisaient pour préparer leurs enseignements et enseigner et leurs sources, la grande majorité ont mentionné que les vidéos (YouTube et d'autres fournisseurs) et les ressources provenant d'Internet comme des animations, des pages Web et des documents sous format DOC et PDF étaient fréquemment utilisées. Même si ces types de ressources ne sont pas toujours des REL au sens technique (p. ex., elles ne sont pas toujours sous licence libre ou adaptables).

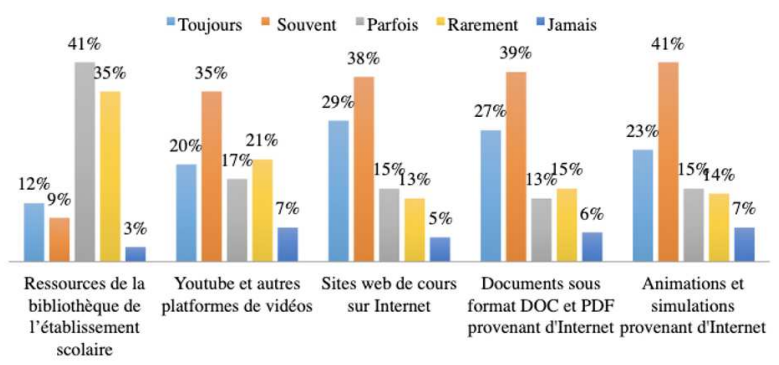

Fig. 11. Fréquence et sources de ressources utilisés par les enseignants. 


\subsubsection{Sensibilisation}

Afin de jauger le niveau de connaissance des REL et les cadres d'octroi de licences et de droits d'auteurs chez notre population d'étude, il a été demandé aux enseignants dans un premier temps de choisir dans la liste suivante l'option qui correspond à leur profil :

- Je n'ai jamais entendu parler des REL

- J'ai entendu parler des REL, mais je ne connais pas grand-chose à leur sujet

- Je connais un peu les REL, mais je ne sais pas comment elles peuvent être utilisées.

- Je connais les REL et certains de leurs cas d'utilisation.

- Je connais très bien les REL et je sais comment elles peuvent être utilisées à des fins d'enseignement.

Le concept des REL est malheureusement méconnu chez les enseignants questionnés. Seulement 25\% confirment connaitre les REL et certains cas de leurs utilisations (Figure 12).

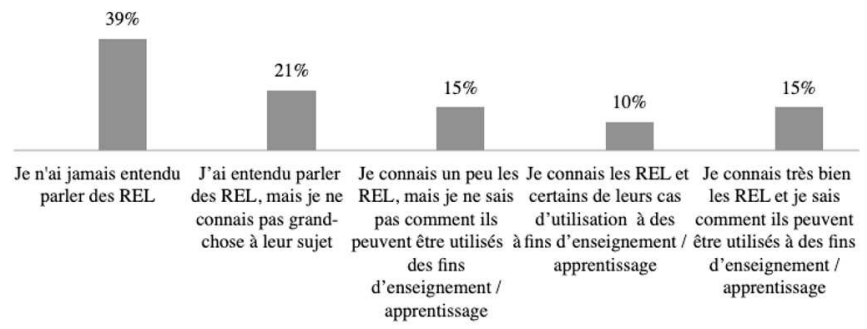

Fig. 12. Niveaux de connaissance des REL.

Une seconde question concernant le niveau de connaissance des cadres d'octroi de licences et de droits d'auteurs a été soumise à la population d'étude en leur demandant de choisir la réponse appropriée (aucune connaissance, vague connaissance, connaissance ou très bonne connaissance) pour chacun des éléments suivants :

- Domaine public

- Droit d'auteur (copyright)

- Creative Commons (ou autre paradigme de licence libre)

Les réponses en Figure 13 montrent que le domaine public reste désormais méconnu chez $68 \%$ de notre population d'étude. 55\% des répondants affirment avoir une connaissance des droits d'auteurs (Copyright). Enfin, 72\% des enseignants questionnés affichent une méconnaissance des licences Créative Commons (ou autre paradigme de licence libre).

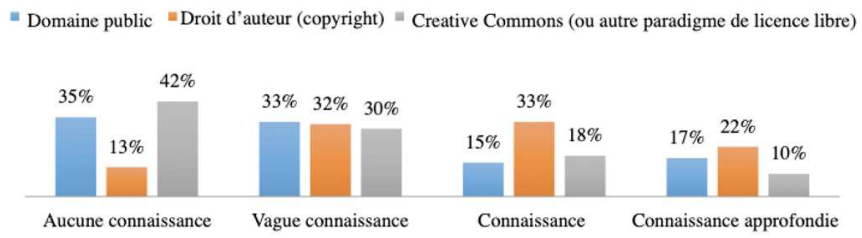

Fig. 13. Niveaux de connaissance des cadres d'octroi de licences et de droits d'auteurs. 


\subsubsection{Capacité}

Deux questions ont été adressées uniquement aux répondants qui ont affiché une connaissance des REL et de leurs cas d'utilisation lors de leur réponse à la question dans la section 4.2.3. Au total, $25 \%$ de notre population d'étude sont concernés comme présenté dans la Figure 11. La première question concerne la disposition des enseignants d'un savoir-faire pour trouver les REL sur Internet via les moteurs de recherche, dans les archives ouvertes, les bibliothèques numériques, les portails spécialisés de REL et les catalogues de REL

Les réponses recueillies (Figure 14) montrent que 60\% des répondants confirment avoir l'expertise suffisante pour trouver des REL sur Internet via les moteurs de recherche, $15 \%$ sont capables de les trouver dans les archives ouvertes, $17 \%$ sont capables de trouver les REL dans les bibliothèques numériques et $20 \%$ sont capables de les trouver dans les portails spécialisés des REL.

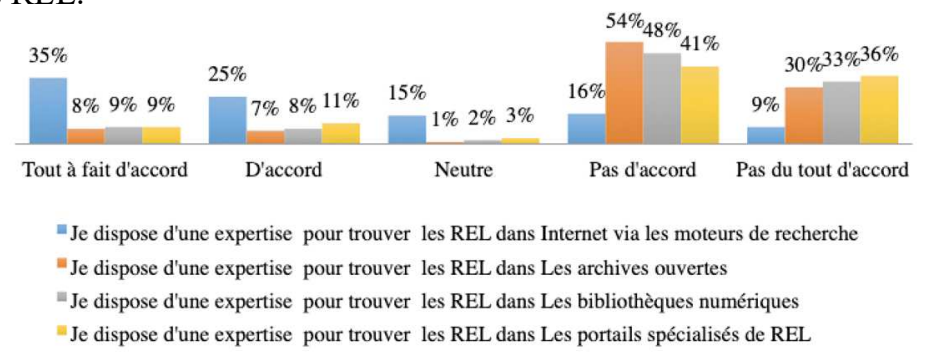

Fig. 14. Niveaux d'expertise pour trouver les REL.

La deuxième question porte sur disposition des enseignants d'un savoir-faire pour l'évaluation de la qualité des REL trouvées, leur adaptation pour qu'elles répondent aux besoins d'enseignement et enfin leur création. Les résultats montrent que sur les $25 \%$ de notre population globale ayant affiché une connaissance des REL et de leurs cas d'utilisation, $56 \%$ déclarent avoir le savoir-faire nécessaire pour évaluer la qualité des REL, 55\% confirment avoir l'expertise pour adapter les REL pour qu'elles s'adaptent aux besoins d'enseignement et $51 \%$ sont capables de créer des REL.

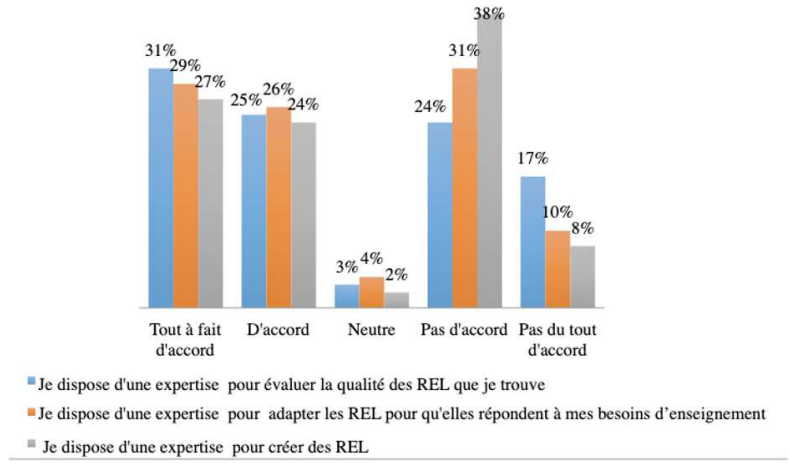

Fig. 15. Niveaux d'expertise pour évaluer, adapter et créer les REL.

\subsubsection{Disponibilité}

Notre objectif dans cette section est d'explorer la disponibilité des REL dans le contexte de l'enseignement scolaire marocain (primaire, secondaire collégial et qualifiant). Une question ouverte a été adressée à notre population d'étude sur les portails et sites web qu'ils fréquentent le plus pour s'approvisionner en ressources d'enseignement/ apprentissage. Ensuite, nous avons procédé à une revue documentaire de l'ensemble de sites web très cités 
par les répondants afin d'identifier les types de ressources qu'elles contiennent et les licences auxquelles elles sont assujetties.

Au total, 24 sites web couvrant différents niveaux d'enseignement scolaire marocain ont été sélectionnés (Tableau 2). Les ressources publiées sont en majorité sous forme textuelle (format DOC, PDF et HTML), vidéos (hébergées sur YouTube et d'autres fournisseurs). Les manuels scolaires sous format numériques sont quasi inexistants.

Table. 2. Format des ressources publiées sur quelques sites éducatifs marocains.

\begin{tabular}{|c|c|}
\hline Sites web et population visée & Format des ressources publiées \\
\hline $\begin{array}{l}\text { www.albostane.com } \\
\text { Population visée : Elèves (niveau } \\
\text { primaire et secondaire), Enseignants } \\
\text { et Directeurs d'établissements } \\
\text { scolaires. }\end{array}$ & $\begin{array}{l}\text { - Ressource sans aucune indication en matière de droits } \\
\text { d'auteurs. Aucune mention relative aux REL. Une } \\
\text { indication « tous droits réservés » est mentionnée en } \\
\text { bas de la page d'accueil du site web }\end{array}$ \\
\hline $\begin{array}{l}\text { www.9rayti.ma } \\
\text { Population visée : Elèves (niveau } \\
\text { secondaire collégial et qualifiant) et } \\
\text { Bacheliers (orientation) }\end{array}$ & $\begin{array}{l}\text { - Ressource sans aucune indication en matière de droits } \\
\text { d'auteurs. Aucune mention relative aux REL. Une } \\
\text { indication « tous droits réservés » est mentionnée en } \\
\text { bas de la page d'accueil du site web }\end{array}$ \\
\hline $\begin{array}{l}\text { www.kezakoo.com } \\
\text { Population visée : Elèves (niveau } \\
\text { secondaire qualifiant) et Bacheliers } \\
\text { (orientation) }\end{array}$ & $\begin{array}{l}\text { - Ressource sans aucune indication en matière de droits } \\
\text { d'auteurs. Aucune mention relative aux REL. }\end{array}$ \\
\hline $\begin{array}{l}\text { www.philomaroc.net } \\
\text { Population visée : Elèves (niveau } \\
\text { secondaire qualifiant) }\end{array}$ & $\begin{array}{l}\text { - Ressource sans aucune indication en matière de droits } \\
\text { d'auteurs. Aucune mention relative aux REL. }\end{array}$ \\
\hline $\begin{array}{l}\text { www.alloschool.com } \\
\text { Population visée : Elèves (niveau } \\
\text { secondaire qualifiant), Bacheliers } \\
\text { (orientation) et Etudiants en classes } \\
\text { préparatoires aux grandes écoles }\end{array}$ & $\begin{array}{l}\text { - Ressource sans aucune indication en matière de droits } \\
\text { d'auteurs. Aucune mention relative aux REL. Une } \\
\text { indication «tous droits réservés » est mentionnée en } \\
\text { bas de la page d'accueil du site web }\end{array}$ \\
\hline $\begin{array}{l}\text { www.chari.123.ma } \\
\text { Population visée : Elèves (niveau } \\
\text { secondaire qualifiant) }\end{array}$ & $\begin{array}{l}\text { - Ressource sans aucune indication en matière de droits } \\
\text { d'auteurs. Aucune mention relative aux REL. }\end{array}$ \\
\hline $\begin{array}{l}\text { www.bacfacile.com } \\
\text { Population visée : Elèves (niveau } \\
\text { secondaire collégial et qualifiant) et } \\
\text { Bacheliers (orientation) }\end{array}$ & $\begin{array}{l}\text { - Ressource sans aucune indication en matière de droits } \\
\text { d'auteurs. Aucune mention relative aux REL. }\end{array}$ \\
\hline $\begin{array}{l}\text { www.bestcours.net } \\
\text { Population visée : Elèves (niveau } \\
\text { primaire et secondaire), Bacheliers } \\
\text { (orientation) et Enseignants. }\end{array}$ & $\begin{array}{l}\text { - Ressources sans aucune indication en matière de droits } \\
\text { d'auteurs. Aucune mention relative aux REL. Une } \\
\text { indication « tous droits réservés » est mentionnée en bas } \\
\text { de la page d'accueil du site web }\end{array}$ \\
\hline $\begin{array}{l}\text { www.moutamadris.ma } \\
\text { Population visée : Elèves (niveau } \\
\text { primaire et secondaire), Bacheliers } \\
\text { (orientation) et Enseignants. }\end{array}$ & $\begin{array}{l}\text { - Ressources sans aucune indication en matière de droits } \\
\text { d'auteurs. Aucune mention relative aux REL. Une } \\
\text { indication « tous droits réservés » est mentionnée en bas } \\
\text { de la page d'accueil du site web }\end{array}$ \\
\hline $\begin{array}{l}\text { www.adirassa.com } \\
\text { Population visée : Elèves (niveau } \\
\text { primaire et secondaire), Bacheliers } \\
\text { (orientation), Futurs enseignants, } \\
\text { Formation professionnelle et } \\
\text { Enseignants. }\end{array}$ & $\begin{array}{l}\text { - Ressources sans aucune indication en matière de droits } \\
\text { d'auteurs. Aucune mention relative aux REL. Une } \\
\text { indication «tous droits réservés » est mentionnée en bas } \\
\text { de la page d'accueil du site web }\end{array}$ \\
\hline $\begin{array}{l}\text { www.madariss.fr } \\
\text { Population visée : Elèves (niveau } \\
\text { secondaire qualifiant) }\end{array}$ & $\begin{array}{l}\text { - Ressources sans aucune indication en matière de droits } \\
\text { d'auteurs. Aucune mention relative aux REL. }\end{array}$ \\
\hline
\end{tabular}




www.pc1.free.fr
Population visée : Elèves (niveau
secondaire collégial)
secondaire collégial)

Www.pc1.ma

Population visée: Elèves (niveau secondaire collégial et qualifiant) et Enseignants.

https://educa.ma

Population visée: Elèves (niveau primaire) et Enseignants.

https://students.ma

Population visée: Elèves (niveau primaire et secondaire) et Bacheliers (orientation)

https://www.tilmide.com/primary/

Population visée: Elèves (niveau secondaire collégial et qualifiant)

https://www.dorossy.com

Population visée: Elèves (niveau primaire et secondaire), Bacheliers (orientation), Futurs enseignants et Enseignants.

https://www.ostaadi.com

Population visée : Futurs enseignants et Enseignants.

https://www.mostajad.com

Population visée: Elèves (niveau primaire et secondaire), Futurs enseignants, Enseignants et Directeurs d'établissements scolaires.

https://www.taalimpress.info

Population visée : Elèves (niveau primaire), Futurs enseignants et Enseignants.

\section{www.bactv.ma}

Population visée: Elèves (niveau secondaire qualifiant) et Bacheliers (orientation)

https://emadrassa.inwi.ma

Population visée: Elèves (niveau primaire et secondaire) et Bacheliers (orientation)

https://annajah.ma

Population visée: Elèves (niveau secondaire qualifiant) et Bacheliers (orientation)

https://alistic.net

Population visée: Elèves (niveau primaire et secondaire) et Etudiants en Brevet de technicien supérieur
- Ressources sans aucune indication en matière de droits d'auteurs. Aucune mention relative aux REL. Une indication « tous droits réservés » est mentionnée en bas de la page d'accueil du site web

- Ressources sans aucune indication en matière de droits d'auteurs. Aucune mention relative aux REL. Une indication « tous droits réservés » est mentionnée en bas de la page d'accueil du site web

- Ressources sans aucune indication en matière de droits d'auteurs. Aucune mention relative aux REL.

- Ressources sans aucune indication en matière de droits d'auteurs. Aucune mention relative aux REL.

- Ressources sans aucune indication en matière de droits d'auteurs. Aucune mention relative aux REL. Une indication « tous droits réservés » est mentionnée en bas de la page d'accueil du site web

- Ressources sans aucune indication en matière de droits d'auteurs. Aucune mention relative aux REL. Une indication « tous droits réservés » est mentionnée en bas de la page d'accueil du site web

- Ressources sans aucune indication en matière de droits d'auteurs. Aucune mention relative aux REL. Une indication « tous droits réservés » est mentionnée en bas de la page d'accueil du site web

- Ressources sans aucune indication en matière de droits d'auteurs. Aucune mention relative aux REL.

- Ressources sans aucune indication en matière de droits d'auteurs. Aucune mention relative aux REL. Une indication « tous droits réservés » est mentionnée en bas de la page d'accueil du site web

- Ressources sans aucune indication en matière de droits d'auteurs. Aucune mention relative aux REL. Une indication « tous droits réservés » est mentionnée en bas de la page d'accueil du site web

- Ressources sans aucune indication en matière de droits d'auteurs. Aucune mention relative aux REL. Une indication « tous droits réservés » est mentionnée en bas de la page d'accueil du site web

- Ressources sans aucune indication en matière de droits d'auteurs. Aucune mention relative aux REL. Une indication « tous droits réservés » est mentionnée en bas de la page d'accueil du site web

Les ressources publiées sur ce site web sont mises à disposition selon les termes de la Licence Créative Commons 
Nous avons aussi mené une revue globale du portail national «ww.taalimtice.ma » mis en place par le Ministère de l'Education Nationale afin de diffuser les ressources numériques éducatives au profit des enseignants marocains. Les résultats montrent que seulement 65 REL figurent parmi les 3239 ressources diffusées sur ce portail comme présenté dans le graphe suivant.

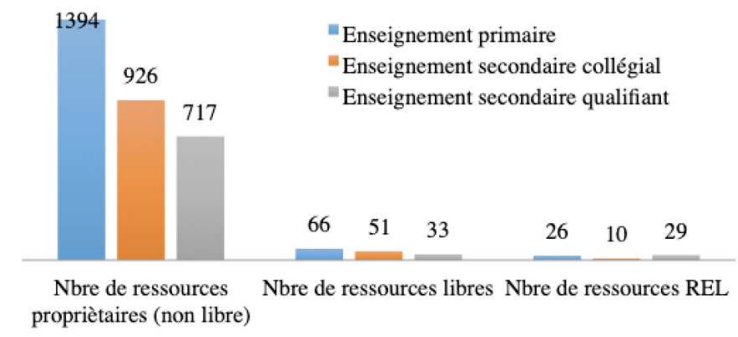

Fig. 16. Format des ressources numériques éducatives publiées dans le portail «taalimtice.ma » selon le niveau enseigné.

\section{Discussion}

Cette étude visait l'étude de la disposition des enseignants marocains à l'adoption des REL via les six facteurs du modèle pyramidal d'adoption des REL présenté dans la figure 1 . Au niveau du premier facteur du modèle, l'accès aux équipements et infrastructures technologiques, notre enquête démontre que les enseignants sont généralement bien équipés en outils informatiques. Concernant la connexion à Internet, les enseignants se connectent majoritairement depuis leur domicile (84\%). La connexion depuis une connexion fournie par l'établissement scolaire reste marginale (21\%); les autres lieux, tels que les cafés, les lieux publics et autres aux alentours représentent 54\%. Par ailleurs, 84\% des répondants se connectent à Internet de façon régulière. Ces chiffres confirment que le facteur d'accès aux équipements et infrastructures technologiques ne constitue aucunement un obstacle aux usages du numérique.

Le deuxième facteur du modèle pyramidal concerne les permissions d'usage et de sélection des REL et les politiques institutionnelles qui les régissent. $81 \%$ des répondants déclarent avoir le plein contrôle sur les décisions relatives à la sélection et l'usage des ressources dans leurs pratiques d'enseignement. Concernant les déterminants potentiels de sélection des ressources du cours. Les enseignants reconnaissent que la richesse du contenu et le format sont les principaux déterminants de sélection des ressources dans leurs pratiques d'enseignement.

$\mathrm{Au}$ regard du troisième facteur, le niveau de connaissance des REL et des licences et des droits d'auteurs est faible chez notre échantillon d'étude. $25 \%$ déclarent avoir des connaissances sur les REL. De nombreux enseignants ne connaissent pas la valeur ajoutée des REL par rapport aux formats de partage qu'ils pratiquent [34]. 72\% des enquêtés affichent une méconnaissance des termes des licences Créative Commons. Plusieurs études ont signalé un tel obstacle auprès des enseignants $[6,31,35]$. Devant un tel constat, il est donc primordial de repenser les programmes de formation continue des enseignants afin qu'ils comprennent des modules sur les concepts des REL et des cadres d'octroi de licences et de droits d'auteurs. De nombreuses études révèlent que l'introduction des concepts des REL aux futurs enseignants pendant leur formation initiale pourrait conduire à un intérêt, une compréhension et une probabilité accrus d'usage des REL [36-38].

Le quatrième facteur est relatif à la disposition des enseignants d'un savoir-faire pour trouver les REL, les évaluer, les adapter et enfin les créer. Seulement, $25 \%$ de notre population globale ayant affiché une connaissance des REL et de leurs cas d'utilisation (Figure 12) sont 
concernés par ce facteur. Les réponses recueillies (Figure 13) montrent que la localisation des REL est parmi les obstacles prédominants chez les participants. Dans ce sens, le développement professionnel visant à accroître la sensibilisation aux sources de dépôt des REL et l'élaboration de listes de ressources REL propres à chaque discipline sont vivement recommandés afin d'accroître l'utilisation globale des REL.

Le cinquième facteur concernant la disponibilité des REL a été approché via une revue d'un ensemble de sites web marocains de soutien scolaire en majorité développé par des enseignants marocains et du portail "taalimtice.ma" affilié au Ministère de l'Education Nationale. Un premier constat révèle une dynamique au sein de la communauté d'enseignants en matière de partage des ressources au profit des enseignants et des élèves. Un deuxième constat concerne l'absence totale de mentions relatives aux droits d'auteurs ou licences d'utilisation dans les ressources qui sont publiées. En effet, sur les 24 sites web consultés, un seul site fait référence aux licences Créative Commons. Sur les 24 sites web consultés, quinze d'entre eux présentent, en bas de leur page d'accueil web, une indication «tous droits réservés », cependant aucun détail n'est fourni concernant les modalités de cette indication. Aucun manuel scolaire ne figure parmi les ressources publiées sur les sites visités.

Le portail national «www.taalimtice.ma » mis en place par le Ministère de l'Education Nationale compte 150 ressources libres et 65 REL parmi les 3239 ressources qui sont diffusées. Bien que le Ministère de l'Education National marocain ait formulé des ambitions sur la promotion de l'usage des REL, l'étude du cinquième facteur montre que la culture des REL est malheureusement à un stade embryonnaire à la fois chez la communauté d'enseignants et les organismes institutionnels marocains.

Le modèle pyramidal de Cox et Trotter, présenté dans la figure 1 au regard de l'adoption des REL repose sur l'idée que la volonté d'adopter ce type de ressources se manifeste lorsque tous les facteurs sous-jacents sont remplis ce qui n'est malheureusement pas le cas dans notre étude.

\section{Limites et recherches futures}

Cette étude a été réalisée sur un échantillon limité de la population des enseignants. Les résultats de cette étude peuvent être utilisés pour éclairer la pratique d'adoption des REL, mais ils ne sont pas applicables à la population globale des enseignants marocains. Envisager la participation d'un échantillon plus représentatif de l'ensemble de la population des enseignants marocaine serait souhaitable dans des recherches futures.

Pour améliorer la compréhension du sujet d'adoption des REL, il est recommandé dans des recherches futures de procéder à des études qualitatives portant sur des cas concrets où les enseignants sont engagés dans l'adoption des REL dans leurs pratiques d'enseignement. Cela pourrait permettre d'obtenir des informations détaillées sur les facteurs et la séquence de la pyramide d'adoption des REL dans différents contextes.

Bien que la plupart de nos conclusions soient confirmées par des études similaires, des recherches supplémentaires sont nécessaires pour étayer ces conclusions avant qu'elles soient généralisées à l'échelle nationale.

\section{Conclusion}

L'usage, la production et la diffusion des REL constituent un mouvement mondial dans le monde de l'éducation, auquel chaque enseignant peut contribuer à sa mesure. Les résultats de cette enquête révèlent un niveau faible de connaissance et d'usage des (REL) et des cadres d'octroi de licences et de droits d'auteurs chez les enseignants. 
Dans la même lignée, la politique institutionnelle concernant ce sujet est encore à un stade embryonnaire. Sur la base de ces résultats, il est recommandé d'établir un plan d'action institutionnel qui vise la promotion de la culture d'adoption des REL au sein de la communauté éducative marocaine à travers un ensemble d'actions à savoir la mise en place des programmes de formation continues et initiales sur l'adoption des REL, les campagnes de sensibilisation et la valorisation institutionnelle de l'usage des REL.uations should be centred and should be numbered with the number on the right-hand side.

\section{Références}

1. UNESCO. (2012). Déclaration de Paris. Congrès national sur les ressources éducatives libres (REL) 2012 Unesco, Paris, 20-22 juin 2012. Récupéré de : www.unesco.org/new/fileadmin/MULTIMEDIA/HQ/CI/CI/pdf/Events/French_Pa ris_OER_Declaration.pdf

2. Unesco (2002). Forum sur l'impact des didacticiels libres pour l'enseignement supérieur dans les pays en développement : rapport final. Récupéré de : http://unesdoc.unesco.org/ images/0012/001285/128515f.pdf

3. Willey (2014). Future of OER. (2014). Iterating toward openness. Récupéré de : http://opencontent.org/blog/archives/3619

4. Belikov, O., \& Bodily, R. (2016). Incentives and barriers to OER adoption: A qualitative analysis of faculty perceptions. Open Praxis, 8(3), 235-246. Récupéré de: http://dx.doi.org/10.5944/openpraxis.8.3.308

5. Dhanarajan, G. \& Porter, D. (Eds.) (2013). Open Educational Resources: An Asian Perspective. Vancouver: Commonwealth of Learning.

6. Jhangiani, R., Pitt, R., Hendricks, C., Key, J., \& Lalonde, C. (2016). Exploring Faculty Use of Open Educational Resources at British Columbia Post-Secondary Institutions. BCcampus Research Report. Victoria, BC: BCcampus. Récupéré de : http://bccampus.ca/files/2016/01/BCFacultyU-seOfOER_final.pdf

7. Clements, K., \& Pawlowski, J. (2012). User-oriented quality for OER: understanding teachers' views on re-use, quality, and trust. Journal of Computer Assisted Learning, 28(1), 4-14. https://doi. org/10.1111/j.1365-2729.2011.00450.

8. UNESCO (2019). Recommandation sur les ressources éducatives libres (REL). Récupéré de : http://portal.unesco.org/fr/ev.php-

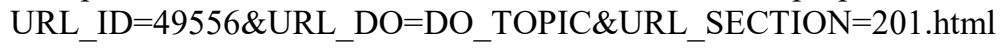

9. Rolfe, V. (2012). Open Educational Resources: Staff attitudes and awareness. Research in Learning Technology, 20:14395. Récupéré de : https://www.dora.dmu.ac.uk/handle/2086/6188

10. Reed, P. (2012). Awareness, attitudes and participation of teaching staff towards the open content movement in one university. Research in Learning Technology, 20. http://dx.doi.org/10.3402/rlt. v20i0.18520

11. Lesko, I. (2013). The use and production of OER \& OCW in teaching in South African higher education institutions (Case study). Open Praxis, 5(2), 102-121. https://doi.org/10.5944/openprax- is.5.2.52

12. Mtebe, J. S., \& Raisamo, R. (2014). Investigating perceived barriers to the use of open educational resources in higher education in Tanzania. The International Review of Research in Open and Distributed Learning, 15(2). http://dx.doi.org/10.19173/irrodl.v15i2.1803 
13. Cox, G., \& Trotter, H. (2016). Institutional culture and OER policy: How structure, culture, and agency mediate OER policy potential in South African universities. The International Review of Research in Open and Distributed Learning, 17(5). https://doi.org/10.19173/irrodl.v17i5.2523

14. Hatakka, M. (2009). Build it and they will come? Inhibiting factors for reuse of open content in developing countries. Electronic Journal on Information Systems in Developing Countries, 37(5), 1-16.

15. Wolfenden, F., Buckler, A., \& Keraro, F. (2012). OER adaptation and reuse across cultural contexts in Sub Saharan Africa: Lessons from TESSA (Teacher Education in Sub Saharan Africa). Journal of Interactive Media in Education, 2012(1), p.Art. 3. http://doi.org/10.5334/2012-03

16. Samzugi, A. S., \& Mwinyimbegu, C. M. (2013). Accessibility of Open Educational Resources for Distance Education Learners: The Case of The Open University of Tanzania. HURIA: Journal of The Open University of Tanzania, 14, 76-88.

17. Baas, M, Admiraal, W \& van den Berg, E. (2019). Teachers' Adoption of Open Educational Resources in Higher Education. Journal of Interactive Media in Education. DOI: https://doi.org/10.5334/jime.510

18. Cox, G. \& Trotter, H. (2017). Factors shaping lecturers' adoption of OER at three South African universities. In C. Hodgkinson-Williams \& P. B. Arinto (Eds.) Adoption and impact of OER in the Global South (pp. 287-347). Récupéré de: https://doi. org/10.5281/zenodo.601935

19. Pegler, C. (2012). Herzberg, hygiene and the motivation to reuse: towards a three-factor theory to explain motivation to share and use OER. Journal of Interactive Media in Education, 2012(1). http://doi.org/10.5334/2012-04

20. Willems, J. \& Bossu, C. (2012). Equity considerations for open educational resources in the glocalization of education. Distance Education, 33(2), 185-199. http://dx.doi.org/10.1080/01587919.2 012.692051

21. Abeywardena, I. S., Dhanarajan, G., \& Chan, C. S. (2012). Searching and Locating OER: Barriers to the Wider Adoption of OER for Teaching in Asia. In Proceedings from the Regional Symposium on Open Educational Resources: An Asian Perspective on Policy and Practices, 19-21 September 2012. Penang: IDRC.

22. CERI/OECD (Centre for Educational Research and Innovation / Organisation for Economic Cooperation and Development) (2007). Giving knowledge for free: The emergence of Open Educational Resources. Récupéré de : http://www.oecd.org/edu/ceri/38654317.pdf

23. Ngimwa, P. (2010). OER Readiness in Africa: A report submitted to the OLnet Project, August 2010. Barcelona: OLnet.

24. Schuwer, R \& Janssen, B. (2018). Adoption of Sharing and Reuse of Open Resources by Educators in Higher Education Institutions in the Netherlands: A Qualitative Research of Practices, Motives, and Con- ditions. The International Review of Research in Open and Distributed Learning, 19(3). DOI: https://doi. org/10.19173/irrodl.v19i3.3390

25. Allen, I. E., \& Seaman, J. (2014). Opening the Curriculum: Open Education Resources in U.S. Higher Education, 2014. Report of the Babson Survey Research Group and The William and Flora Hewlett Foundation. Récupéré de : http://www.onlinelearningsurvey.com/reports/openingthecur- riculum2014.pdf 
26. Beetham, H., Falconer, I., McGill, L. \& Littlejohn, A. (2012). Open Practices: Briefing Paper.

de: https://oersynth.pbworks.com/w/page/51668352/OpenPracticesBriefing

Récupéré

27. Davis, H. C., Carr, L., Hey, J. M. N., Howard, Y., Millard, D., Morris, D., \& White, S. (2010). Bootstrapping a culture of sharing to facilitate Open Educational Resources. IEEE Transactions on Learning Technologies, 2(10), 1-14.

28. Kursun, E., Cagiltay, K., \& Can, G. (2014). An investigation of faculty perspectives on barriers, in- centives, and benefits of the OER movement in Turkey. The International Review of Research in Open and Distributed Learning, 15(6), 13-32. Récupéré de : http://dx.doi.org/10.19173/irrodl.v15i6.1914

29. Maslow, A. H. (1943). A Theory of Human Motivation. Psychological Review, 50, 370396. McGill, L., Falconer, I., Dempster, J. A., Littlejohn, A. \& Beetham, H. (2013). Journeys to Open Educational Practice: UKOER/SCORE Review Final Report. London: JISC. Open Praxis, vol. 9 issue 2, April-June 2017, pp. 151-171

30. Cox, G., \& Trotter, H. (2016). Institutional culture and OER policy: How structure, culture, and agency mediate OER policy potential in South African universities. The International Review of Research in Open and Distributed Learning, 17(5). https://doi.org/10.19173/irrodl.v17i5.2523

31. Cox, G., \& Trotter, H. (2017). An OER framework, heuristic and lens: Tools for understanding lecturers' adoption of OER. Open Praxis, 9(2), 151-171.

32. Farrow, R, Perryman, LA, de los Arcos, B, Weller, M \& Pitt, R. (2016). OER Hub Researcher Pack, 16 December. Récupéré de: https://oerhub.pressbooks.com

33. Rapport de l'eCampus Ontario, 2018. Connaissance et utilisation des ressources éducatives libres (REL) en Ontario : Étude préliminaire des perspectives des enseignants au postsecondaire. Récupéré de : https://www.ecampusontario.ca/wpcontent/uploads/2018/10/eCampusOntario_September2018_ResearchReport_FR_V2.p $\mathrm{df}$

34. Delos Arcos, B., Cannell, P., \& McIlwhan, R. (2016). Awareness of open educational resources (OER) and open educational practice (OEP) in Scottish higher education institutions. OEPS Interim Report. Récupéré de: https://www.slideshare.net/OEPScotland/awareness-of-oer-and-oep- in-scottish-highereducation-institutions-survey-results

35. Allen, I., \& Seaman, J. (2016). Opening the textbook: Educational resources in U.S. higher education, 2015-16. Thornhill: Babson Survey Research Group. Récupéré de : https://www.onlinelearningsurvey.com/reports/openingthetextbook2016.pdf

36. Misra, P. (2014). Online training of teachers using OER: Promises and potential strategies. Open Praxis, 6(4), 375-385. http://dx.doi.org/10.5944/openpraxis.6.4.155

37. Ramírez-Montoya, M-S., Mena, J., \& Rodríguez-Arroyo, J. (2017). In-service Teachers' self-perceptions of digital competence and OER use as determined by a XMOOC training $\begin{array}{lllll}\text { course. Computers in Human 356- } & \text { Behavior, }\end{array}$ 364. https://doi.org/10.1016/j.chb.2017.09.010

38. Tur, G., Urbina, S., \& Moreno, J. (2016). From OER to open ed perceptions of student teachers. BRAIN: Broad Research in Artificial Intelligence and Neuroscience, 7(2), 3440. Récupéré de : http://www.edusoft.ro/brain/index.php/brain/article/view/594. 